\title{
Entorno tecnológico y composición cualitativa del tejido empresarial en la UE-15*
}

\author{
Ana Millán \\ Universitat Autònoma de Barcelona \\ José María Millán \\ Concepción Román \\ Universidad de Huelva
}

\section{Resumen}

Este trabajo analiza el papel de la actividad innovadora y el entorno tecnológico de un país en la decisión de ser empleador (autoempleado con asalariados) frente a la alternativa de ser trabajador autónomo (autoempleado sin asalariados). Para ello, se estiman modelos de elección discreta con variable dependiente binaria utilizando los microdatos del Panel de Hogares de la Unión Europea (PHOGUE). Como medidas de innovación, se utilizan diferentes indicadores agregados a nivel de país que miden tanto el esfuerzo tecnológico (gasto en actividades de $I+D$ y número de empleados en actividades de $I+D$ ) como los resultados de la actividad científico-técnica (solicitudes de patentes ante la Oficina Europea de Patentes -EPO- y la Oficina de Patentes y Marcas de Estados Unidos -USPTO-). Nuestros resultados sugieren que las actividades y esfuerzos en materia de innovación afectan a la composición relativa del tejido empresarial en favor de los empleadores frente a los trabajadores autónomos.

Palabras clave: innovación, entorno tecnológico, empleadores, autónomos, emprendimiento, Europa.

Clasificación JEL: E02, J23, J24, J28, L25, L26, M51, O30, O52.

\begin{abstract}
This work analyses the role of innovation and technological environment at the country level in the individual decision to be an employer (self-employed with employees) against the alternative of being an own-account worker (self-employed without employees). To this end, we estimate binary discrete choice models, using microdata drawn from the European Community Household Panel (ECHP). As measures of innovation, we use different aggregated indicators at the country level aimed at capturing both technological effort $(R \& D$ expenditures and employment share of high-tech and knowledge-intensive sectors) and results of scientifictechnical activity (number of patent applications to the European Patent Office -EPO- and to the United States Patent and Trademark Office-USPTO-). We find strong support for a positive relationship between these indicators of the technological environment and the relative weight of employers on total selfemployment.
\end{abstract}

* Los autores quisieran expresar su gratitud al editor de Cuadernos Económicos de ICE, Gustavo Nombela, a las editoras del monográfico María Engracia Rochina y Amparo Sanchis (Universidad de Valencia), a Fernando Millán (Universidad de Sevilla) y a un evaluador anónimo por sus comentarios y sugerencias. Asimismo, agradecen la financiación del Ministerio de Economía y Competitividad (Ana Millán al proyecto ECO2013-48496-C4-4-R, y José María Millán y Concepción Román al proyecto ECO2013-43526-R) para la realización de este trabajo. Todos los errores son, por supuesto, responsabilidad de los autores. 
Keywords: innovation, technological environment, employers, own-account workers, entrepreneurship, Europe.

JEL classification: E02, J23, J24, J28, L25, L26, M51, O30, O52.

\section{Introducción}

Tras más de seis años del comienzo de la peor crisis económica y financiera de las últimas cinco décadas, la economía global empieza a recuperarse lentamente. Si bien la recuperación económica parece afianzarse, el desafío es asegurar que esta se acompañe de un crecimiento sostenido del empleo. En este sentido, la estrategia Europa $2020^{1}$ establece como uno de sus objetivos prioritarios que la tasa de empleo de la población de entre 20 y 64 años alcance el 75 por 100 . Los datos correspondientes al cuarto trimestre de 2014 sitúan dicha tasa de empleo en el 70,1 por 100 en la UE15 (Eurostat, Labour Force Survey). Comparando este dato con el correspondiente al cuarto trimestre de 2009, que situaba esta proporción en el 69,7 por 100, podemos observar cómo tras cinco años el dato solo ha mejorado en 0,4 puntos porcentuales. Aun considerando el impacto que la crisis ha tenido en esta progresión, es bastante improbable que se alcance el objetivo planteado para 2020 (European Commission, 2015). Además, el dato agregado del último trimestre de 2014 recoge realidades a nivel nacional muy dispares. Por una parte, incluye situaciones como la de Alemania que, con una tasa de empleo de la banda de edad considerada del 77,9 por 100, ya ha alcanzado su objetivo nacional, marcado en el 77 por 100; o Suecia que con un 79,7 por 100 se posiciona muy cerca de su objetivo nacional ${ }^{2}$, establecido en el 80 por 100. Pero por otra parte, incluye también a países como Grecia y España que, con unas tasas de empleo de 53,4 por 100 y 60,8 por 100 , respectivamente, están aún muy alejados de los objetivos nacionales, situados respectivamente en 70 por 100 y 74 por 100 .

Del análisis anterior parece desprenderse que aún queda camino por recorrer en relación al objetivo prioritario en materia de empleo establecido por la estrategia Europa 2020. En consecuencia, el debate acerca de cómo mejorar las tasas de empleo de las economías más afectadas por la crisis económica sigue estando en el

\footnotetext{
${ }^{1}$ Europa 2020 es la estrategia de crecimiento de la Unión Europea (UE) para la presente década aprobada por el Consejo Europeo el 17 de junio de 2010. Esta estrategia establece para el año 2020 ambiciosos objetivos en materia de empleo, I+D, cambio climático y energía, educación y pobreza y exclusión social. Así, dicha estrategia persigue: (i) aumentar la tasa de empleo de la población de entre 20 y 64 años hasta al menos el 75 por 100; (ii) aumentar la inversión en I+D hasta alcanzar el 3 por 100 del PIB; (iii) reducir las emisiones de gases de efecto invernadero un 20 por 100 (con respecto a los niveles de 1990); (iv) conseguir que un 20 por 100 del consumo final de energía sea de energías renovables; (v) aumentar un 20 por 100 la eficiencia energética; (vi) situar la tasa de abandono escolar prematuro por debajo del 10 por 100; (vii) lograr que al menos un 40 por 100 de las personas de 30 a 34 años de edad completen estudios de nivel terciario; (viii) reducir al menos en 20 millones el número de personas en situación o riesgo de pobreza y exclusión social (European Commission, 2015).

${ }^{2}$ Los objetivos nacionales se corresponden con los planteados en los National Reform Programmes (NRP) en abril de 2014, teniendo en cuenta las diferentes situaciones y circunstancias de cada país miembro, así como su compromiso con el objetivo comunitario.
} 
corazón de la política económica europea y cualquier contribución al respecto merece especial atención. En este sentido, hay un consenso casi unánime entre académicos de que la actividad empresarial es el motor principal del crecimiento económico, de la competitividad en el mercado global y de la propia creación de empleo (véanse, por ejemplo, los trabajos de Carree y Thurik, 2008; Thurik et al., 2008; o Parker, 2009). Asimismo, la propia estrategia Europa 2020 destaca el papel fundamental del emprendimiento en estos procesos (European Commission, 2010, 2015).

Precisamente en esta línea, la UE plantea en su Plan de Acción sobre Emprendimiento 2020 una serie de medidas de apoyo con el objetivo de aumentar el número de emprendedores en Europa (European Commission, 2013) ${ }^{3}$. Sin embargo, dado el carácter heterogéneo de este grupo, donde solo una minoría contrata a otros trabajadores (Congregado et al., 2010; Cowling et al., 2004) y/o crea empresas que generan innovación y riqueza (Blanchflower, 2004; Shane, 2009), aumentar cuantitativamente el tejido empresarial no tiene por qué ser necesariamente mejor. Es decir, si el objetivo es favorecer los procesos anteriormente mencionados de generación de riqueza y empleo, quizás habría que concentrar los esfuerzos en el desarrollo de medidas que ayuden a aquellos individuos con verdadera vocación emprendedora (que no abandonen el trabajo por cuenta propia cuando surjan oportunidades en el empleo asalariado o cuando desaparezcan los incentivos a la entrada; véase Lucas, 1978), de forma que se incremente la calidad (y no solo la cantidad) del tejido empresarial. Nos estamos refiriendo a favorecer al emprendedor por motivo oportunidad frente a aquel que lo es por motivo necesidad (Reynolds et al., 2002), al empresario innovador frente al imitador (Schumpeter, 1912), o al empleador con asalariados frente al trabajador autónomo (Román et al., 2013; J. M. Millán et al., 2014a). Precisamente esta última distinción cobra especial relevancia en el contexto actual pues nos permite separar a aquellos emprendedores que más contribuyen a los procesos de generación de empleo de aquellos que tan solo se emplean a sí mismos ${ }^{4}$.

La composición cuantitativa y cualitativa del tejido empresarial depende de múltiples factores, de forma que la contribución al empleo del emprendimiento no es un fenómeno aleatorio. En este sentido, los factores a nivel individual parecen insuficientes para explicar por qué el tejido empresarial de algunas economías ha

\footnotetext{
${ }^{3}$ El Plan de Acción sobre Emprendimiento 2020 es un programa de acciones conjuntas para favorecer el desarrollo del emprendimiento en Europa, cuyo objetivo es facilitar la creación de empresas y crear un entorno mucho más favorable para los emprendedores. Se basa en los siguientes tres pilares: (i) desarrollar la educación y formación en materia de emprendimiento; (ii) crear un entorno empresarial favorable, eliminando barreras estructurales y prestando apoyo en las fases cruciales del ciclo vital de la empresa; y (iii) establecer modelos y fomentar el emprendimiento entre grupos específicos, como desempleados, mujeres e inmigrantes (European Commission, 2013).

${ }^{4}$ La literatura también considera la distinción entre empleadores y autónomos como una proxy de la distinción entre verdaderos emprendedores y otras figuras diversas como los profesionales liberales, los autoempleados por motivo necesidad e incluso los falsos autónomos (KUHN, 2000; ROMÁN et al., 2011, 2013). Si bien la asociación entre empleadores y verdaderos emprendedores admite cierto debate, la contribución de los empleadores al empleo y la facilidad para identificar a este grupo en las encuestas de población no admite discusión alguna. Véase MILLÁN et al. (2008) para una revisión de las fuentes de información disponibles para el estudio del emprendimiento en España.
} 
resistido mejor que otros las turbulencias propias del período recesivo reciente (mientras que otras destruyeron empleo de forma alarmante). Por tanto, en aras de entender estas diferencias, analizar algunos factores idiosincráticos de país se revela como un objetivo prioritario. Así, algunas instituciones del mercado de trabajo como las políticas activas de empleo o la legislación sobre la protección del empleo parecen tener una influencia relevante en el peso relativo de los empleadores -frente a los trabajadores autónomos- sobre el total de autoempleados (Román et al., 2011, 2013; A. Millán et al.,2013). Del mismo modo, el nivel de formación de la propia sociedad puede moldear tanto la oferta como la demanda en los mercados de productos y servicios, afectando también a la composición del tejido empresarial (J. M. Millán et al., 2014c).

De la misma forma, cabe esperar que el carácter más o menos innovador de una determinada economía tenga reservado un papel fundamental en esta ecuación. En este sentido, la cantidad de recursos destinados a actividades de I+D en una economía (ya sea en términos monetarios o de empleo) -así como los propios resultados de esta inversión (en términos de innovaciones concretas)- deben redundar positivamente en los procesos de creación y expansión de conocimiento (knowledge spillovers), incrementando el stock de capital tecnológico, y aumentando en definitiva las oportunidades empresariales (véanse Audretsch, 1995, y Kirzner, 1997). Además, un avanzado entorno tecnológico puede provocar un efecto de auto selección, de forma que ante un mercado más complejo y turbulento solo los mejores y más preparados decidan aventurarse a emprender, lo que redunda en un tejido empresarial de más calidad y con mayor potencial de generación de empleo (Acs y Audretsch, 2003; Acemoglu et al. 2006; Crifo y Sami, 2008). Algunos estudios empíricos muestran cómo la inversión en I+D está asociada con unas mayores tasas de creación de empresas (Lee et al., 2004; Choi y Phan, 2006) y supervivencia empresarial (Congregado et al., 2011; Van Stel et al., 2014). Sin embargo, el papel concreto de este entorno innovador y tecnológico en la composición cualitativa del tejido empresarial y su contribución al empleo, esto es, en el peso relativo de los empleadores -frente a los trabajadores autónomos- sobre el total de autoempleados, permanece aún inexplorado por la comunidad académica.

Cabe destacar en este punto, que precisamente otro de los objetivos prioritarios de la estrategia Europa 2020 establece que la inversión en I+D alcance el 3 por 100 del PIB. Los últimos datos disponibles, correspondientes a 2013, sitúan dicho indicador en el 2,09 por 100 para la UE-15. Al igual que hemos mencionado en el caso de la tasa de empleo, este dato agregado oculta la disparidad existente entre economías como Dinamarca, que con un 3,06 por 100 de su PIB dedicado a I+D está ya por encima de su objetivo nacional, establecido en el 3 por 100, y Portugal, que con solo un 1,36 por 100 aún está a mitad de camino de alcanzar su objetivo nacional (2,7 por 100). España, con un dato para 2013 del 1,24 por 100, también se encuentra lejos del 2 por 100 planteado.

Por tanto, a menos de 5 años de alcanzar el 2020, la distancia por recorrer tanto en términos de inversión en I+D como de empleo es significativa. Y por ello, las 
medidas destinadas a alcanzar estos objetivos deben tener en cuenta las fuertes interrelaciones existentes entre los propios objetivos. En este sentido, el fenómeno del emprendimiento debe ser el vehículo transmisor entre la inversión en I+D y la generación de empleo. Nuestra premisa es precisamente que los esfuerzos y resultados en materia de innovación existentes en una economía deben generar una composición cualitativa del tejido empresarial más favorable a los procesos de creación de empleo. Por tanto, una apuesta decidida por mejorar el entorno tecnológico y de innovación de un país debería acercarnos de forma simultánea a la consecución de los objetivos de la estrategia Europa 2020 tanto en materia de innovación como de empleo.

En línea con la discusión anterior, el objetivo de este trabajo es precisamente contrastar si el carácter innovador de una economía (medido a través de diferentes indicadores agregados de esfuerzo tecnológico y de resultados de la actividad científico-técnica a nivel de país) influye en la composición cualitativa del tejido empresarial de la UE-15 y, en particular, en el peso relativo de los empleadores (autoempleados con asalariados) y los trabajadores autónomos (autoempleados sin asalariados) sobre el total de autoempleados de un país.

Para llevar a cabo esta tarea, se estiman modelos de elección discreta con variable dependiente binaria utilizando los microdatos del Panel de Hogares de la Unión Europea (PHOGUE) para el período 1994-2001. Nuestros resultados sugieren que las actividades y esfuerzos en materia de innovación en un país afectan a la composición relativa del tejido empresarial en favor de los empleadores frente a los trabajadores autónomos.

El resto del trabajo se organiza de la siguiente manera: la sección 2 describe la base de datos, el modo en que se ha diseñado la muestra y las variables utilizadas en el análisis empírico. La sección 3 presenta el marco econométrico, mientras que la sección 4 presenta y comenta los resultados. Finalmente, la sección 5 concluye.

\section{Datos, diseño muestral y variables}

\subsection{Fuente de datos: Panel de Hogares de la Unión Europea (PHOGUE)}

Como ya hemos mencionado, en este trabajo utilizamos los microdatos del Panel de Hogares de la Unión Europea (PHOGUE) ${ }^{5}$. El PHOGUE, diseñado y coordinado por EUROSTAT, es un panel fijo a nivel de la UE- $15^{6}$ que recoge información estadística sobre 76.500 hogares (equivalentes a unas 155.000 personas) durante el período 1994-2001. El mismo cuestionario ha sido utilizado en todos los países, lo que genera información armonizada y directamente comparable.

\footnotetext{
${ }^{5}$ Los datos del PHOGUE se utilizan con el permiso de Eurostat (contrato ECHP/2006/09 con la Universidad de Huelva).

${ }^{6}$ Se ha excluido a Luxemburgo y Suecia, pues la información recogida en el PHOGUE presenta muchos valores missing en variables relevantes para la realización de este análisis.
} 


\subsection{Diseño muestral}

A los individuos incluidos en el PHOGUE se les pregunta acerca de: (i) su situación en la actividad principal (empleado asalariado, autoempleado, ayuda familiar, desempleado o inactivo); y (ii) el número de asalariados regulares en el centro de trabajo en el empleo actual. A partir de esta información podemos identificar en nuestra base de datos a los empleadores (autoempleados con asalariados) y los trabajadores autónomos (autoempleados sin asalariados). La muestra final incluye 56.300 observaciones correspondientes a 16.191 hombres y mujeres autoempleados con y sin asalariados con edades comprendidas entre los 18 y los 65 años ${ }^{7}$.

\subsection{Variables explicativas principales: variables de innovación}

Como hemos discutido en la sección introductoria, el protagonismo fundamental de las actividades tecnológicas en el éxito empresarial, y por ende, en el desarrollo económico urge a los países a impulsar la innovación en sus economías nacionales. En este sentido, la complejidad del cambio tecnológico como fenómeno sistémico nos obliga a considerar un conjunto de variables o indicadores encargados de medir las distintas dimensiones y aspectos relevantes de las actividades tecnológicas. La forma convencional de presentar los indicadores que se manejan en el ámbito de la ciencia y la tecnología distingue entre indicadores de esfuerzo tecnológico e indicadores de resultados de la actividad científico-técnica ${ }^{8}$. Por ello, con el objetivo de recoger la presencia y la apuesta por las actividades de innovación en cada una de las economías analizadas, los siguientes indicadores conforman las variables explicativas principales en nuestro análisis:

1. Gasto en actividades de I+D de las administraciones públicas, el sector privado y los centros de enseñanza superior, expresados como porcentaje del PIB.

2. Porcentaje del empleo en industrias de alta tecnología y servicios intensivos en conocimiento de alta tecnología, sobre el empleo total en el país.

3. Solicitudes de patentes presentadas ante la Oficina Europea de Patentes (EPO) por cada millón de habitantes.

4. Solicitudes de patentes presentadas ante la Oficina de Patentes y Marcas de EEUU (USPTO) por cada millón de habitantes.

${ }^{7}$ Aunque el diseño del PHOGUE garantiza su representatividad para los países considerados, la selección muestral que se lleva a cabo en este estudio puede condicionar dicha representatividad. Es necesario, por tanto, matizar que los resultados presentados están condicionados por esta selección muestral.

${ }^{8}$ También se recogen una serie de indicadores compuestos que tratan de analizar múltiples dimensiones en el terreno de la innovación, como el Índice Europeo de Innovación (IEI) y el Índice de Adelanto Tecnológico (IAT). Ninguno de estos índices ha podido utilizarse en este trabajo, pues empezaron a publicarse a partir de 2001. 
Así, nuestro primer indicador (gasto en actividades de I+D) es una medida de esfuerzo global que trata de cuantificar los recursos que se dedican a tareas de investigación y desarrollo, y representa la aproximación más inmediata para medir los esfuerzos que se llevan a cabo en el terreno tecnológico. Su simplicidad conceptual y el hecho de que se refieran al núcleo básico de la formación del conocimiento científico y técnico lo han convertido en el indicador más habitual para resumir los esfuerzos que se llevan a cabo en este ámbito.

A efectos comparativos y con objeto de evitar la distorsión que pueda derivarse de la utilización de unidades monetarias (una menor retribución al personal investigador supondría unos menores gastos en I+D, que no significarían un menor esfuerzo en este terreno), añadimos un segundo indicador proporcionando los datos en términos de volumen de empleo dedicado a estas tareas (número de empleados en actividades de I+D por cada 100 ocupados).

Los indicadores tercero y cuarto (solicitudes de patentes ante la EPO y la USPTO) atienden a los resultados obtenidos a través de los esfuerzos desplegados en el terreno tecnológico. Así, las patentes son una valiosa fuente de información sobre el desarrollo tecnológico generado por las empresas o centros de investigación aplicada. En esa línea, las solicitudes de patentes nos muestran la capacidad inventiva de cada país para desarrollar novedades técnicas susceptibles de ser explotadas comercialmente? .

\subsection{Variables de control}

Nuestras estimaciones incluyen un conjunto de variables de control que sabemos que influyen en la elección de ocupación y el desempeño empresarial ${ }^{10}$. Estas variables son las siguientes: (i) género (muchos estudios previos señalan que las mujeres empresarias operan a menor escala, muestran un mayor porcentaje de fracaso y menores tasas de creación de empleo; véanse por ejemplo Boden y Nucci, 2000, y Burke et al., 2002); (ii) edad y edad al cuadrado (la edad favorece la creación de redes y contactos y la identificación de mejores oportunidades de negocio; Rees y Shah, 1986; Taylor, 1996; Clark y Drinkwater, 1998); (iii) vivir en pareja (estar casado se asocia a la disponibilidad de mayores niveles de capital tanto financiero como social y, por tanto, mayores niveles de entrada y éxito en la actividad empresarial; véanse Shane, 2003, pp. 67-68; Parker, 2009, p. 132, y Román et al., 2013); (iv) número de hijos en el hogar (la evidencia empírica sobre el efecto de los hijos en el desempeño empresarial es mixta; véase Williams, 2004); (v) estado de salud (el autoempleo ofrece una mayor flexibilidad en la organización del puesto de trabajo que, en muchas ocasiones, es necesaria para aquellos individuos con peor estado

\footnotetext{
${ }^{9}$ Una descripción detallada de estas variables está disponible en el Anexo (Cuadro 2). Además, el Anexo incluye los valores medios de las variables de innovación por países (Cuadro 3) y sus correlaciones (Cuadro 4).

${ }^{10}$ Una descripción detallada de estas variables de control está disponible en el Anexo (Cuadro 2).
} 
de salud; véanse Quinn, 1980 y Parker, 2009, pp. 133-134); (vi) capacidad para llegar a fin de mes (que puede ser indicativa de la situación financiera del negocio y que se espera que afecte positivamente a las decisiones de contratación, tal y como recogen A. Millán et al., 2015); (vii) educación (el capital humano obtenido a través de la educación es uno de los determinantes más relevantes del éxito empresarial, independientemente de si se utilizan los ingresos, la supervivencia, la contribución al empleo o el crecimiento de las ventas para evaluar el desempeño; véase el resumen de Van der Sluis et al., 2008); (viii) horas de trabajo (que podrían interpretarse como una proxy de la demanda a la que se enfrenta el negocio como sugieren J.M. Millán et al., 2013); y (ix) una medida de la duración en el empleo actual (que trata de recoger la existencia de un efecto aprendizaje en la gestión del negocio; véanse, por ejemplo, los trabajos de Taylor, 2004; Van Praag, 2003, y J.M. Millán et al., 2012, 2014a, 2014 b , 2014c). Finalmente, también se incluyen en este análisis dummies de sector y de país. Para una revisión más extensiva del efecto de estas y otras variables sobre el desempeño empresarial, véanse Parker, 2009, y J.M. Millán et al., 2012.

\section{Marco econométrico}

Esta sección describe brevemente el marco econométrico utilizado en este trabajo. En particular, utilizamos modelos de elección discreta con variable dependiente binaria. Nuestra variable dependiente $Y_{i, t}$ toma el valor 1 si el individuo es observado como empleador en el período $t$ y el valor 0 si el individuo es observado como trabajador autónomo en el mismo período. Esta variable $Y_{i, t}$ observada está relacionada con una variable latente $Y_{i, t}^{*}$ (no observable) que puede ser interpretada como la diferencia entre la utilidad que reporta al individuo ser empleador y la utilidad que le reporta ser autónomo $\left(U_{i, t}^{E}-U_{i, t}^{A}\right)$. De este modo, un individuo será observado en el período $t$ como empleador (autónomo) si la utilidad que le genera ser empleador $\left(U_{i, t}^{E}\right)$ excede (no supera) aquella que le genera ser autónomo $\left(U_{i, t}^{A}\right)$. En términos más formales:

$$
\begin{array}{lll}
Y_{i, t}=1 & \text { si } & Y_{i, t}^{*}=U_{i, t}^{E}-U_{i, t}^{A}>0 \\
Y_{i, t}=0 & \text { si } & Y_{i, t}^{*}=U_{i, t}^{E}-U_{i, t}^{A} \leq 0
\end{array}
$$

Como es habitual en estos modelos, la probabilidad de ser observado en alguna de las dos situaciones laborales consideradas depende de un conjunto de características individuales y del entorno que definen las utilidades de ambas alternativas. En particular, la probabilidad de que el individuo $i$ sea empleador en el período $t$ puede ser descrita en los siguientes términos:

$$
\begin{gathered}
\operatorname{Pr}\left(Y_{i, t}=1\right)=\operatorname{Pr}\left(Y_{i, t}^{*}=U_{i, t}^{E}-U_{i, t}^{A}>0\right)= \\
=\operatorname{Pr}\left(\beta^{\prime} X_{i, t}+u_{i}+\varepsilon_{i, t}>0\right)=\operatorname{Pr}\left(\varepsilon_{i, t}<\beta^{\prime} X_{i, t}+u_{i}\right)=F\left(\beta^{\prime} X_{i, t}+u_{i}\right)
\end{gathered}
$$


donde el vector $X_{i, t}$ representa características individuales (demográficas, educativas y del trabajo) y del entorno (dummies de país y variables de innovación), $\beta$ es el vector de coeficientes a estimar sobre la base de nuestras observaciones, $u_{i}$ es el término que captura la heterogeneidad inobservable o efecto específico individual (que es invariante en el tiempo) ${ }^{11}, \varepsilon_{i, t}$ es el término de error aleatorio, y la función $\mathrm{F}(\cdot)$ sigue una distribución logística:

$$
F(z)=\frac{\exp (z)}{1+\exp (z)}
$$

\section{Resultados y discusión}

Esta sección presenta los resultados del análisis empírico realizado acerca del papel que tiene el entorno tecnológico en la composición relativa del tejido empresarial en la UE-15 y, en particular, en el peso relativo que tienen los empleadores frente a los trabajadores autónomos en esta composición. La subsección 4.1 se centra en los resultados principales, relativos a las variables de innovación, mientras que la subsección 4.2 se centra en los resultados que emergen de la inclusión de las variables de control. Estos resultados se presentan en el Cuadro 1, que incluye cinco especificaciones. La especificación I incluye como variables explicativas las características demográficas, la educación, las características del trabajo y las dummies de sector y de país. Las especificaciones II-V incluyen cada una de las cuatro variables de innovación consideradas en el análisis. En este sentido, el Cuadro 4 incluido en el apéndice nos muestra las elevadas correlaciones existentes entre nuestras cuatro medidas de innovación, lo que justifica incluir cada una de ellas en una especificación independiente. Así, la especificación II estudia el papel del gasto en actividades de I+D, la especificación III cubre el efecto del volumen de empleo dedicado a estas tareas, la especificación IV se centra en el impacto de las solicitudes de patentes ante la EPO y, finalmente, la especificación V analiza el rol que tienen las solicitudes de patentes ante la USPTO ${ }^{12}$.

Los resultados se presentan del siguiente modo. Cada especificación muestra su correspondiente probabilidad de ser empleador -frente a la alternativa de ser

\footnotetext{
11 Supongamos dos observaciones $y_{i, 1}$ e $y_{i, 2}$ del mismo individuo i correspondientes a dos momentos del tiempo diferentes. En consecuencia, los términos $u_{i, 1}$ y $u_{i, 2}$ no estarán distribuidos de manera independiente puesto que pertenecen al mismo individuo sino que, por el contrario, tenderán a ser muy similares. Excluyendo estos términos en la estimación nos llevaría, para todos los individuos en nuestra muestra, a infravalorar la verdadera variación de nuestro término de error $\varepsilon_{i, t}$ y a sobreestimar la significatividad estadística de nuestros coeficientes $\beta$. Esta es la razón por la que incorporamos un término $u_{i}$ que recoge la heterogeneidad inobservable o efecto específico individual. Dado que nuestras estimaciones son realizadas a través de modelos de efectos aleatorios, la distribución de este término se asume como el de una variable aleatoria normal con media 0 y varianza $u_{n}$.

${ }^{12}$ Hemos realizado tests de razón de verosimilitud para contrastar si la inclusión de las variables de innovación en las especificaciones II a V mejora el poder explicativo de estas especificaciones sobre la I, obteniendo resultados positivos con un nivel de significatividad del 1 por 100. Por brevedad, no se muestran los resultados de estos tests, estando disponibles bajo petición.
} 
autónomo- para los valores medios, tanto de variables explicativas continuas como discretas. Además, cada especificación se presenta en un formato de dos columnas, en el que se muestran los efectos marginales (y no los coeficientes) junto con los estadísticos $t$. Nótese que un efecto marginal mayor que cero implica un efecto positivo sobre la probabilidad de ser empleador, o lo que es lo mismo, un efecto negativo en la probabilidad de ser autónomo. Al final de cada especificación hemos incorporado los resultados del test de razón de verosimilitud que compara la bondad de ajuste del modelo que incorpora el efecto específico individual (datos de panel) con el modelo que no lo incluye (modelo agrupado) ${ }^{13}$.

\subsection{Resultados principales: variables de innovación}

Nuestros resultados indican que los cuatro indicadores de innovación utilizados, esto es, (i) el gasto en actividades de I+D; (ii) el volumen de empleo dedicado a estas tareas; (iii) las solicitudes de patentes ante la EPO; y (iv) ante la USPTO influyen positivamente en la probabilidad de ser empleador -frente a la alternativa de ser autónomo-. En otras palabras, el entorno tecnológico parece estar asociado positivamente con el tamaño medio de los negocios de un país. Las especificaciones II a V del Cuadro 1 nos muestran estos resultados.

En particular, vemos cómo las probabilidades predichas de ser empleador se encuentran en torno al 50 por 100 y cómo estas probabilidades aumentan significativamente al aumentar cualquiera de nuestras variables de innovación ${ }^{14}$. Para una mejor comprensión de la magnitud de los efectos marginales, nuestras variables de innovación han sido reescaladas de forma que el efecto marginal asociado al gasto en actividades de I+D refleja cómo la probabilidad de ser empleador varía cuando el gasto como porcentaje del PIB aumenta en un 0,1 por $100^{15}$. Así pues, la especificación II nos muestra cómo la probabilidad de ser empleador aumenta casi en 5 puntos porcentuales cuando el gasto en actividades de I+D como porcentaje del PIB aumenta en un 0,1 por 100. De forma similar, los efectos marginales asociados con el empleo en industrias de alta tecnología recogen cómo la probabilidad de ser empleador varía cuando el porcentaje de empleo que se dedica a estas actividades sobre el empleo total en el país aumenta en un 0,1 por $100^{16}$. En este caso, la

\footnotetext{
13 Para las cinco especificaciones, rechazamos la hipótesis nula de la ausencia de efectos específicos individuales $(\rho=0)$, por lo que el modelo de datos de panel es preferible al modelo agrupado.

${ }^{14}$ La robustez de estos resultados se mantiene cuando desagregamos por sectores de financiación (o ejecución) las variables sobre el gasto en actividades de I+D y las solicitudes de patentes ante la EPO, distinguiendo entre administraciones públicas, educación superior (universidades), y empresas. Los resultados relativos a esta desagregación se omiten por brevedad y están disponibles bajo petición.

15 Presentar los efectos marginales sobre la base de incrementos del 1 por 100 en esta variable es bastante irrealista, teniendo en cuenta que el valor medio para el gasto en actividades de I+D como porcentaje del PIB es del 1,2 por 100 en los países considerados en el análisis.

16 Del mismo modo, presentar efectos marginales sobre la base de incrementos del 1 por 100 en la población empleada dedicada a estas actividades de alta tecnología no parece una escala adecuada pues el valor medio para los países considerados ronda el 3,5 por 100 .
} 


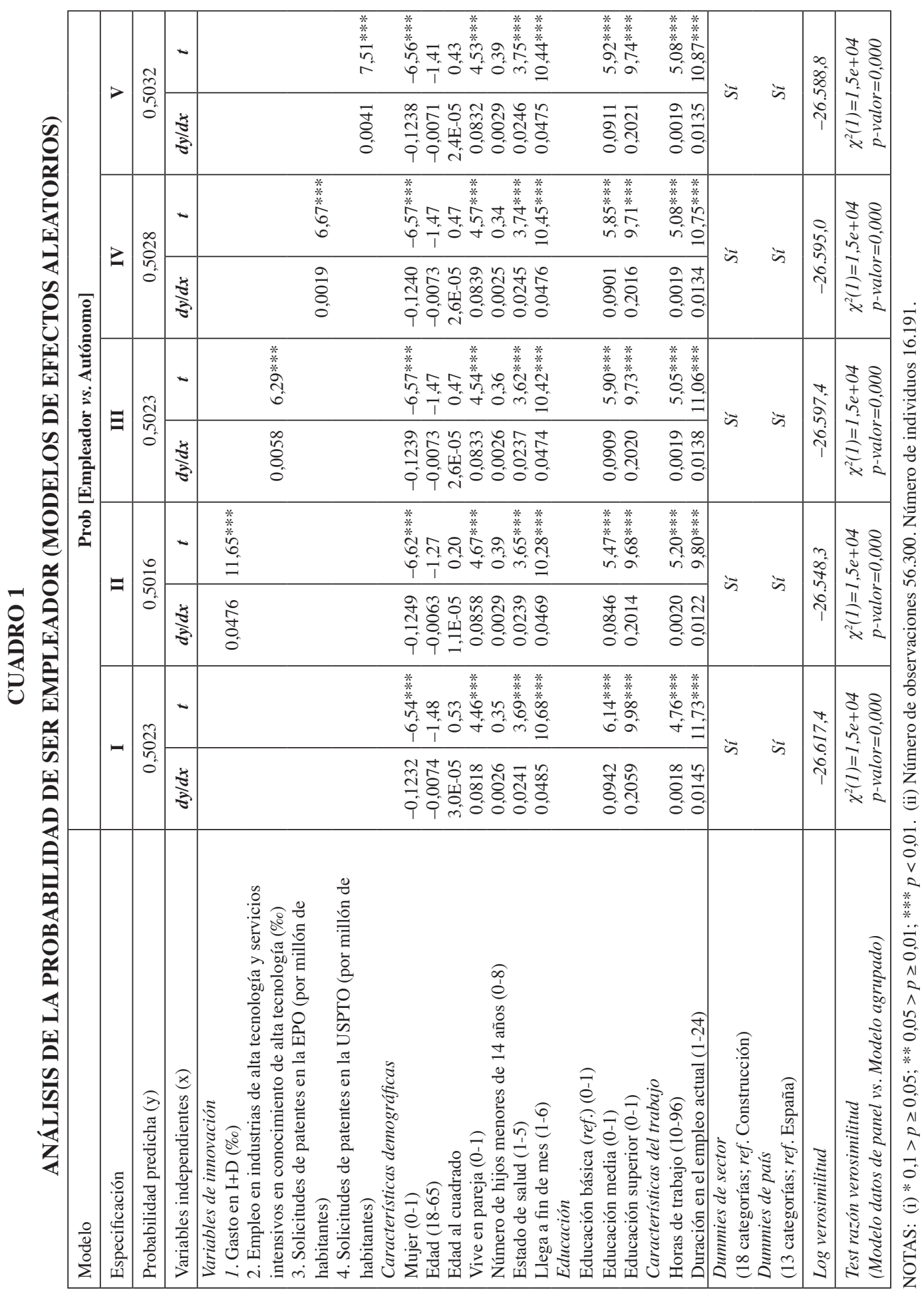


especificación III muestra cómo la probabilidad de ser empleador aumenta casi en 0,6 por 100 puntos porcentuales por cada aumento del 0,1 por 100 en el porcentaje de empleo en actividades de innovación sobre el empleo total en el país. En relación a las solicitudes de patentes, tanto en la EPO como en la USPTO, los efectos marginales pueden interpretarse como el efecto de una solicitud adicional por cada millón de habitantes. En este sentido, las especificaciones IV y V indican que la probabilidad de ser empleador aumenta casi en 0,2 por 100 puntos porcentuales por cada solicitud adicional de patente en la EPO por cada millón de habitantes y en más del 0,4 por 100 por cada solicitud adicional de patente en la USPTO.

En resumen, nuestros resultados principales sustentan la proposición de que el esfuerzo global en materia de innovación afecta a la composición relativa del tejido empresarial en favor de los empleadores frente a los trabajadores autónomos. Por tanto, de cara a la consecución de los objetivos planteados tanto en materia de empleo como de I+D dentro de la estrategia Europa 2020, parece recomendable que las diferentes administraciones promuevan mayores esfuerzos en términos de innovación.

\subsection{Otros resultados: variables de control}

Los efectos más relevantes de las variables de control pueden resumirse del siguiente modo. En cuanto a las características demográficas, observamos en primer lugar como las mujeres presentan una probabilidad de ser empleadoras 12 puntos porcentuales inferior a la de los varones. Además, aquellos autoempleados que viven en pareja presentan una probabilidad de ser empleador unos 8 puntos porcentuales por encima de aquellos que no lo hacen. Variables como el estado de salud o llegar a fin de mes también aumentan las probabilidades de contratación de los autoempleados.

La educación es, sin embargo, la variable con mayor influencia en el peso de empleadores y autónomos. Así, aquellos autoempleados con educación superior muestran una probabilidad de ser empleador 20 puntos por encima de aquellos autoempleados con educación básica. Igualmente, aquellos autoempleados con educación media presentan probabilidades de ser empleador unos 9 puntos por encima de aquellos que solo tienen educación básica. Se confirma, pues, el papel que tiene el capital humano en el éxito de las actividades de emprendimiento, medidas estas en términos de contribución a los procesos de creación de empleo.

Finalmente, vemos que algunas características del trabajo como las horas de trabajo y la propia duración en el empleo actual presentan una relación positiva con la probabilidad de emplear trabajadores por parte de los autoempleados. 


\section{Conclusiones}

Existe un consenso casi unánime entre académicos (y políticos) de que recuperar los datos de empleo previos a la crisis económica pasa por reconocer como elemento clave el papel del emprendimiento. Sin embargo, la respuesta del tejido empresarial a dicha crisis no ha sido uniforme entre países. Así, el tejido empresarial de algunas economías ha resistido mejor esta fase recesiva del ciclo, lo que apunta a la necesidad de considerar los factores idiosincráticos de país a la hora de identificar los determinantes de la composición cualitativa del tejido empresarial en cuanto a su capacidad para generar empleo. La literatura reciente ya destaca el papel relevante que tienen algunas instituciones del mercado de trabajo (políticas activas, legislación sobre la protección del empleo, etcétera) e incluso el nivel de formación de la propia sociedad en esta composición. No obstante, la actividad innovadora de un país también podría contribuir a explicar las diferencias detectadas en la fortaleza de los diferentes tejidos empresariales a la hora de crear empleo.

Partiendo de esta premisa, este trabajo analiza el papel de la actividad innovadora de un país en la proporción de emprendedores que contribuyen a la creación de empleo -frente a aquellos que solo se emplean a sí mismos- con respecto al total de emprendedores en los países de la UE-15. Como medidas de innovación, se utilizan diferentes indicadores agregados a nivel de país de esfuerzo tecnológico y resultados de la actividad científico-técnica.

Nuestros resultados muestran la existencia de una relación positiva y robusta entre las actividades de innovación existentes a nivel de país y la composición cualitativa del tejido empresarial en favor de los empleadores -frente a los trabajadores autónomos-, que podría verse beneficiada de este entorno innovador, y de los procesos asociados de creación, expansión (knowledge spillovers) y comercialización de este conocimiento, aumentando sus posibilidades de identificación y explotación de nuevas oportunidades empresariales.

Estos resultados ilustran la importancia del esfuerzo que realizan las economías en actividades de I+D para los propios procesos de generación de empleo. Parece confirmarse la necesidad de aumentar los recursos destinados a mejorar el entorno tecnológico y de innovación de un país, lo que contrasta con las decisiones tomadas por algunos gobiernos e instituciones, que han decidido reducir significativamente las inversiones en esta materia, como forma de ajustar el gasto en este período recesivo.

\section{Referencias bibliográficas}

[1] ACEMOGLU,D.,AGHION, P. y ZILIBOTTI,Z.(2006). «Distance to frontier, selection, and economic growth». Journal of the European Economic Association 4(1), 37-74.

[2] ACS, Z. J. y AUDRETSCH, D.B. (2003). «Innovation and technological change». en Z.J. Acs y D.B. Audretsch (eds.), Handbook of Entrepreneurship Research, Boston, MA: Kluwer Academic Publishers, 55-79. 
[3] AUDRETSCH, D.B. (1995). Innovation and Industry Evolution. Cambridge, MA: MIT Press.

[4] BLANCHFLOWER,D.G. (2004). «Self-employment: More may not be better». Swedish Economic Policy Review, 11(2), 15-74.

[5] BODEN, R. J. y NUCCI, A. R. (2000). «On the survival prospects of men's and women's new business ventures». Journal of Business Venturing, 15(4), 347-362.

[6] BURKE, A.E., FITZ ROY, F.R. y NOLAN, M.A. (2002). «Self-employment wealth and job creation: The roles of gender, non-pecuniary motivation and entrepreneurial ability». Small Business Economics 19(3), 255-270.

[7] CARREE, M. y THURIK, R. (2008). «The lag structure of the impact of business ownership on economic performance in OECD countries». Small Business Economics 30(1), 101-110.

[8] CHOI, Y.R. y PHAN, P.H. (2006). «The influences of economic and technology policy on the dynamics of new firm formation». Small Business Economics 26(4), 493-503.

[9] CLARK, K. y DRINKWATER, S. (1998). «Ethnicity and self-employment in Britain». Oxford Bulletin of Economics and Statistics 60(3), 383-407.

[10] CONGREGADO, E., GOLPE, A.A. y CARMONA, M. (2010). «Is it a good policy to promote self- employment for job creation? Evidence from Spain». Journal of Policy Modeling, 32(6), 843-864.

[11] CONGREGADO, E., MILLÁN, J.M. y ROMÁN, C. (2011). «El papel de la innovación en la supervivencia de los emprendedores UE-15». Economía Industrial, 382, 119127.

[12] COWLING, M., MITCHELL, P. y TAYLOR, M. (2004). «Job creators». The Manchester School, 72(5), 601-617.

[13] CRIFO, P. y SAMI, H. (2008). «Entrepreneurship turnover and endogenous returns to ability». Economic Modelling, 25(4), 585-604.

[14] EUROPEAN COMMISSION (2015). Smarter, greener, more inclusive? - Indicators to support the Europe 2020 strategy. Luxembourg: Publications Office of the European Union.

[15] EUROPEAN COMMISSION (2013). Entrepreneurship 2020 Action Plan - Reigniting the entrepreneurial spirit in Europe. $\mathrm{COM}(2012) 795$ final, Brussels.

[16] EUROPEAN COMMISSION (2010). Europe 2020-A strategy for smart, sustainable and inclusive growth. $\mathrm{COM(2010)} 2020$ final, Brussels.

[17] KIRZNER, I.M. (1997). «Entrepreneurial discovery and the competitive market process: An Austrian approach». Journal of Economic Literature, 35(1), 60-85.

[18] KUHN, P. (2000). «Editor's note». Labour Economics, 7(5), 463-469.

[19] LEE, S., FLORIDA, R. y ACS, Z. (2004). «Creativity and entrepreneurship: a regional analysis of new firm formation». Regional Studies, 38(8), 879-891.

[20] LUCAS, R.E. (1978). «On the size distribution of business firms». Bell Journal of Economics, 9(2), 508-523.

[21] MILLÁN, A., MILLÁN, J.M., ROMÁN, C. y VAN STEL, A. (2015). «Unraveling the relationship between the business cycle and the own-account worker's decision to hire employees». International Entrepreneurship and Management Journal, 11(2), 331342.

[22] MILLÁN, J.M., CONGREGADO, E. y ROMÁN, C. (2008). «Entrepreneurship analysis from a human population surveys' perspective». En E. Congregado (ed.), Measuring 
Entrepreneurship: Building a Statistical System, Series: International Studies in Entrepreneurship, Springer, vol. 16, 85-112.

[23] MILLÁN, J.M., CONGREGADO, E. y ROMÁN, C. (2012). «Determinants of selfemployment survival in Europe». Small Business Economics, 38(2), 231-258.

[24] MILlÁN, J.M., CONGREGADO, E. y ROMÁN, C. (2014a). «Entrepreneurship persistence with and without personnel: The role of human capital and previous unemployment». International Entrepreneurship and Management Journal, 10(1), 187206.

[25] Millán, J.M., CONGREGADO, E. y ROMÁN, C. (2014b). «Persistence in entrepreneurship and its implications for the European entrepreneurial promotion policy». Journal of Policy Modeling, 36(1), 83-106.

[26] MILLÁN, J.M., CONGREGADO, E., ROMÁN, C., VAN PRAAG, M. y VAN STEL, A. $(2014 \mathrm{c})$. «The value of an educated population for an individual's entrepreneurship success». Journal of Business Venturing, 29(5), 612-632.

[27] MILLÁN, J.M., HESSELS, J., THURIK, R. y AGUADO, R. (2013). «Determinants of job satisfaction: A European comparison of self-employed and paid employees». Small Business Economics, 40(3), 651-670.

[28] PARKER, S.C. (2009). The Economics of Entrepreneurship. Cambridge, UK: Cambridge University Press.

[29] QUINN, J.F. (1980). «Labour-force participation patterns of older self-employed workers». Social Security Bulletin, 43(4), 17-28.

[30] REES, H. y SHAH, A. (1986). «An empirical analysis of self employment in the UK». Journal of Applied Econometrics, 1(1), 95-108.

[31] REYNOLDS, P., CAMP, S.M., BYGRAVE, W.D., AUTIO, E. y HAY, M. (2002). «Global Entrepreneurship Monitor 2001 Executive Report». Babson Park/London: Babson College and London Business School.

[32] ROMÁN, C., CONGREGADO, E. y MILLÁN, J.M. (2011). «Dependent self-employment as a way to evade employment protection legislation». Small Business Economics 37(3), 363-392.

[33] ROMÁN, C., CONGREGADO, E. y MILLÁN, J.M. (2013). «Start-up incentives: entrepreneurship policy or active labour market programme?». Journal of Business Venturing, 28(1), 151-175.

[34] SCHUMPETER, J.A. (1912). Theorie der Wirtschaftlichen Entwicklung (The Theory of Economic Development). Leipzig: Dunker \& Humblot; traducido por Dedvers Opie. Cambridge, MA: Harvard University Press, 1934.

[35] SHANE, S. (2003). A General Theory of Entrepreneurship: the Individual-Opportunity Nexus. Cheltenham, UK: Edward Elgar.

[36] SHANE, S. (2009). «Why encouraging more people to become entrepreneurs is bad public policy». Small Business Economics, 33(2), 141-149.

[37] TAYLOR, M.P. (1996). «Earnings, independence or unemployment: Why become selfemployed?». Oxford Bulletin of Economics and Statistics, 58(2), 253-266.

[38] TAYLOR, M.P. (2004). «Self-employment in Britain: when, who and why?». Swedish Economic Policy Review, 11(2), 139-173.

[39] THURIK, A.R., CARREE, M.A., VAN STEL, A. y AUDRETSCH, D.A. (2008). «Does self-employment reduce unemployment?». Journal of Business Venturing, 23(6), 673686. 
[40] VAN DER SLUIS, J., VAN PRAAG, C.M. y VIJVERBERG, W. (2008). «Education and entrepreneurship selection and performance: A review of the empirical literature». Journal of Economic Surveys, 22(5), 795-841.

[41] VAN PRAAG, C.M. (2003). «Business survival and success of young small business owners». Small Business Economics 21(1), 1-17.

[42] VAN STEL, A., MILLÁN, J.M. y ROMÁN, C. (2014). «Investigating the impact of the technological environment on survival chances of employer entrepreneurs». Small Business Economics, 43(4), 839-855.

[43] WILLIAMS, D.R. (2004). «Effects of childcare activities on the duration of selfemployment in Europe». Entrepreneurship Theory and Practice, 28(5), 467-485. 


\begin{abstract}
ANEXO
CUADRO 2

DESCRIPCIÓN DETALLADA DE LAS VARIABLES UTILIZADAS EN EL ANÁLISIS
\end{abstract}

\begin{tabular}{|c|c|}
\hline Variable & Descripción \\
\hline & Variable dependiente \\
\hline \multirow[t]{2}{*}{ Empleador vs. Autónomo } & $\begin{array}{l}\text { Dummy igual a } 1 \text { para empleadores } \\
\text { (autoempleados con asalariados) y } 0 \text { para } \\
\text { trabajadores autónomos (autoempleados sin } \\
\text { asalariados). }\end{array}$ \\
\hline & Variables independientes \\
\hline \multicolumn{2}{|l|}{ Variables de innovación } \\
\hline 1. Gasto en I+D & $\begin{array}{l}\text { Gastos internos totales en actividades de I+D de } \\
\text { las administraciones públicas, el sector privado } \\
\text { (incluyendo instituciones privadas sin fines } \\
\text { de lucro), y los centros de enseñanza superior, } \\
\text { expresados como porcentaje del PIB. Fuente: } \\
\text { Eurostat. }\end{array}$ \\
\hline $\begin{array}{l}\text { 2. Empleo en industrias de alta tecnología, y } \\
\text { servicios intensivos en conocimiento de alta } \\
\text { tecnología }\end{array}$ & $\begin{array}{l}\text { Porcentaje del empleo en industrias de } \\
\text { alta tecnología y servicios intensivos en } \\
\text { conocimiento de alta tecnología, sobre el empleo } \\
\text { total en el país (Nomenclature of Economic } \\
\text { Activities Rev.1.1): } \\
\text { Industrias de alta tecnología: } \\
\text { DG24.4 Fabricación de productos farmacéuticos } \\
\text { y botánicos. } \\
\text { DL30 Fabricación de maquinaria de oficina, } \\
\text { cálculo y ordenadores. } \\
\text { DL32 Fabricación de aparatos de televisión, } \\
\text { radio y comunicaciones. } \\
\text { DL33 Fabricación de equipo e instrumentos } \\
\text { médico-quirúrgicos, de precisión, óptica y } \\
\text { relojería. } \\
\text { DM35.3 Construcción aeronaútica y espacial. } \\
\text { Servicios intensivos en conocimiento de alta } \\
\text { tecnología: } \\
\text { I64 Servicios de correos y telecomunicaciones. } \\
\text { K72 Equipos informáticos y actividades } \\
\text { relacionadas. } \\
\text { K73 Investigación y desarrollo. } \\
\text { Fuente: Eurostat. }\end{array}$ \\
\hline 3. Solicitudes de patentes en la EPO & $\begin{array}{l}\text { Número de solicitudes de patentes presentadas } \\
\text { ante la Oficina Europea de Patentes (EPO) } \\
\text { procedentes del país, por cada millón de } \\
\text { habitantes. Fuente: Eurostat. }\end{array}$ \\
\hline 4. Solicitudes de patentes en la USPTO & $\begin{array}{l}\text { Número de solicitudes de patentes presentadas } \\
\text { ante la Oficina de Patentes y Marcas de EE.UU. } \\
\text { (USPTO) procedentes del país, por cada millón } \\
\text { de habitantes. Fuente: Eurostat. }\end{array}$ \\
\hline
\end{tabular}




\section{CUADRO 2 (continuación)}

DESCRIPCIÓN DETALLADA DE LAS VARIABLES UTILIZADAS EN EL ANÁLISIS

\begin{tabular}{|c|c|}
\hline Variable & Descripción \\
\hline \multicolumn{2}{|l|}{$\begin{array}{l}\text { Variables de control } \\
\text { Características demográficas }\end{array}$} \\
\hline Mujer & Dummy que toma el valor 1 para mujeres. \\
\hline Edad & Edad del individuo, variando entre 18 y 65 años. \\
\hline Vive en pareja & $\begin{array}{l}\text { Dummy que toma el valor } 1 \text { para individuos que } \\
\text { viven en pareja. }\end{array}$ \\
\hline Número de hijos menores de 14 años & $\begin{array}{l}\text { Número de hijos menores de } 14 \text { años que viven } \\
\text { en el hogar. Esta variable varía entre } 0 \text { y } 8 \text {. }\end{array}$ \\
\hline Estado de salud & $\begin{array}{l}\text { Variable discreta con valores entre } 1 \text { y } 5 \text {, que } \\
\text { captura el estado de salud del individuo. El } 1 \text { se } \\
\text { refiere a individuos con un estado de salud muy } \\
\text { malo y el } 5 \text { a individuos con estado de salud } \\
\text { muy bueno. }\end{array}$ \\
\hline Llega a fin de mes & $\begin{array}{l}\text { Variable discreta con valores entre } 1 \text { y } 6 \text {, que } \\
\text { captura la facilidad o dificultad con que el hogar } \\
\text { llega a fin de mes. El } 1 \text { se refiere a hogares que } \\
\text { llegan a fin de mes con mucha dificultad y el } \\
6 \text { a hogares que llegan a fin de mes con suma } \\
\text { facilidad. }\end{array}$ \\
\hline \multicolumn{2}{|l|}{ Educación } \\
\hline Educación básica (ref.) & $\begin{array}{l}\text { Dummy que toma el valor } 1 \text { para individuos que } \\
\text { no tienen educación secundaria. Se corresponde } \\
\text { con los valores } 0,1 \text { y } 2 \text { de la International } \\
\text { Standard Classification of Education (ISCED). }\end{array}$ \\
\hline Educación media & $\begin{array}{l}\text { Dummy que toma el valor } 1 \text { para individuos que } \\
\text { tienen educación secundaria, pero no estudios } \\
\text { superiores. Se corresponde con el valor } 3 \text { de la } \\
\text { ISCED. }\end{array}$ \\
\hline Educación superior & $\begin{array}{l}\text { Dummy que toma el valor } 1 \text { para individuos que } \\
\text { tienen estudios superiores. Se corresponde con } \\
\text { los valores } 5,6 \text { y } 7 \text { de la ISCED. }\end{array}$ \\
\hline \multicolumn{2}{|l|}{ Características del trabajo } \\
\hline Horas de trabajo & $\begin{array}{l}\text { Horas de trabajo semanales. Esta variable varía } \\
\text { entre } 10 \text { y } 96 \text { horas semanales }\end{array}$ \\
\hline Duración en el empleo actual & $\begin{array}{l}\text { Número de años en el presente trabajo. Esta } \\
\text { variable varía entre } 1 \text { y } 24 \text { años. }\end{array}$ \\
\hline
\end{tabular}




\section{CUADRO 2 (continuación)}

DESCRIPCIÓN DETALLADA DE LAS VARIABLES UTILIZADAS EN EL ANÁLISIS

\begin{tabular}{|c|c|}
\hline Variable & Descripción \\
\hline Dummies de sector & $\begin{array}{l}18 \text { dummies que toman el valor } 1 \text { para aquellos } \\
\text { individuos cuyo código de actividad principal de } \\
\text { su negocio se corresponde con las siguientes } \\
\text { A+B (ref.) Agricultura, ganadería, caza y } \\
\text { silvicultura + Pesca. } \\
\text { C+E Industrias extractivas; producción y } \\
\text { distribución de energía eléctrica, gas y agua. } \\
\text { DA Industrias de productos alimenticios, } \\
\text { bebidas y tabaco. } \\
\text { DB+DC Industria textil; industria de la } \\
\text { confección, peletería y cuero. } \\
\text { DD+DE Industria de la madera, corcho y } \\
\text { papel; edición, artes gráficas y reproducción de } \\
\text { soportes grabados. } \\
\text { DF-DI Coquerías, refino de petróleo y } \\
\text { tratamiento de combustibles nucleares; industria } \\
\text { química; fabricación de productos de caucho } \\
\text { y materias plásticas; fabricación de otros } \\
\text { productos minerales no metálicos. } \\
\text { DL-DN Otras manufacturas. } \\
\text { F Construcción } \\
\text { G Comercio; reparación de vehículos de } \\
\text { motor, motocicletas y ciclomotores y artículos } \\
\text { personales y de uso doméstico. } \\
\text { H Hostelería. } \\
\text { I Transporte, almacenamiento y comunicaciones. } \\
\text { J Intermediación financiera. } \\
\text { K Actividades inmobiliarias y de alquiler; } \\
\text { servicios empresariales. } \\
\text { L Administración pública, defensa y seguridad } \\
\text { social obligatoria. } \\
\text { M Educación. } \\
\text { N Actividades sanitarias y veterinarias; servicios } \\
\text { sociales. } \\
\text { O-Q Otras actividades sociales y de servicios } \\
\text { prestados a la comunidad; servicios personales; } \\
\text { hogares que emplean personal doméstico; } \\
\text { organismos extraterritoriales. }\end{array}$ \\
\hline Dummies de país & $\begin{array}{l}13 \text { dummies que toman el valor } 1 \text { para aquellos } \\
\text { individuos que viven en el correspondiente } \\
\text { país de la UE-15: Alemania, Austria, Bélgica, } \\
\text { Dinamarca, España (ref.), Finlandia, Francia, } \\
\text { Grecia, Holanda, Irlanda, Italia, Portugal y el } \\
\text { Reino Unido. }\end{array}$ \\
\hline
\end{tabular}




\section{CUADRO 3}

VALORES MEDIOS DE LAS VARIABLES DE INNOVACIÓN POR PAÍSES (PERÍODO 1994-2001)

\begin{tabular}{|l|c|c|c|c|}
\hline $\begin{array}{c}\text { Variables de } \\
\text { innovación }\end{array}$ & 1. Gasto en I+D & $\begin{array}{c}\text { 2. Empleo en } \\
\text { industrias de } \\
\text { alta tecnología } \\
\text { y servicios } \\
\text { intensivos en } \\
\text { conocimiento de } \\
\text { alta tecnología }\end{array}$ & $\begin{array}{c}\text { 3. Solicitudes de } \\
\text { patentes en la } \\
\text { EPO }\end{array}$ & $\begin{array}{c}\text { 4. Solicitudes de } \\
\text { patentes en la } \\
\text { USPTO }\end{array}$ \\
\hline Alemania & $2,30 \%$ & $4,46 \%$ & 217,7 & 139,5 \\
\hline Austria & $1,76 \%$ & $4,48 \%$ & 117,1 & 71,3 \\
\hline Bélgica & $1,85 \%$ & $4,40 \%$ & 105,7 & 73,1 \\
\hline Dinamarca & $2,06 \%$ & $5,17 \%$ & 134,8 & 94,6 \\
\hline España & $0,85 \%$ & $2,63 \%$ & 15,1 & 8,2 \\
\hline Finlandia & $2,81 \%$ & $6,01 \%$ & 210,6 & 173,3 \\
\hline Francia & $2,22 \%$ & $5,19 \%$ & 105,3 & 69,6 \\
\hline Grecia & $0,52 \%$ & $1,66 \%$ & 4,5 & 2,1 \\
\hline Holanda & $1,92 \%$ & $4,59 \%$ & 164,4 & 95,6 \\
\hline Irlanda & $1,22 \%$ & $6,13 \%$ & 43,4 & 41,7 \\
\hline Italia & $1,03 \%$ & $3,74 \%$ & 56,8 & 31,3 \\
\hline Portugal & $0,65 \%$ & $2,01 \%$ & 2,7 & 1,3 \\
\hline Reino Unido & $1,83 \%$ & $5,41 \%$ & 83,0 & 68,6 \\
\hline
\end{tabular}

FUENTE: Eurostat.

\section{CUADRO 4}

\section{CORRELACIONES DE LAS VARIABLES DE INNOVACIÓN}

\begin{tabular}{|l|c|c|c|}
\hline \multicolumn{1}{|c|}{ Variables de innovación } & 1. & $\mathbf{2 .}$ & $\mathbf{3 .}$ \\
\hline Gasto en I+D & & & \\
\hline $\begin{array}{l}\text { Empleo en industrias de alta tecnología y servicios } \\
\text { intensivos en conocimiento de alta tecnología }\end{array}$ & 0,8110 & & \\
\hline Solicitudes de patentes en la EPO & 0,9309 & 0,7593 & \\
\hline Solicitudes de patentes en la USPTO & 0,9461 & 0,7729 & 0,9705 \\
\hline
\end{tabular}

FUENTE: Eurostat. 